\title{
Idiopathic Chondrolysis of the Hip ( ICH): Report of three Cases
}

\author{
C Dechosilpa, MD, P Mulpruek, MD, MCh Orth, P Woratanarat, MD, PhD (Clinical Epidemiology) \\ P Thiabratana, MD* \\ Department of Orthopaedics, Ramathibodi Hospital, Mahidol University, Bangkok, Thailand \\ *Department of Orthopaedic Surgery, Bhumibol Adulyadej Hospital, Bangkok, Thailand
}

\begin{abstract}
Idiopathic Chondrolysis of the Hip (ICH) is a rare condition, occurring mostly in black female adolescence. It is characterized by the rapidly progressive destruction of articular cartilage in the hip joint resulting in premature degeneration and subsequent joint arthrosis. We report three cases of ICH: a 13-year old boy presented with left knee pain, an 11-year old girl with right hip pain and a 12-year old girl with right thigh pain. All of them had the same characteristic radiographic findings. The initial treatment was started conservatively. Surgical treatment was performed in one patient in order to confirm diagnosis and correct deformity.
\end{abstract}

Key Words:

Chondrolysis, Idiopathic; Hip; Paediatric; Stiffness

\section{INTRODUCTION}

Hip joint chondrolysis was first reported by Waldenstrom in which referred to the condition of acute cartilage necrosis associated with slipped capital femoral epiphysis (SCFE), trauma, infection, and prolonged immobilization. Jones in 1971, described a series of hip joint chondrolysis in adolescent black females. In seven out of nine cases the causes could not be identified. In 1975, Duncan ${ }^{1}$ reported five patients with the same condition and used the term "Idiopathic Chondrolysis of the Hip" to describe this phenomenon.

ICH is characterized by the rapidly progressive destruction of articular cartilage in the hip joint, in the absence of known aetiologies ${ }^{1-5}$. The diagnosis is made by exclusion of other hip diseases with similar symptoms and signs. Most patients presented with hip, groin or knee pain, with limping or stiffness of the affected hip. Laboratory tests are usually normal. Concentric narrowing (less than $3 \mathrm{~mm}$ ) of joint space without frank osteophyte formation in conjunction with periarticular osteopenia is the hallmark radiographic finding $2,3,5$. The natural history of ICH with or without treatment is unpredictable.
Three cases of ICH are reported.

\section{CASE REPORTS}

Case 1

An obese 13-year old boy presented with left knee pain of six months duration. There was no history of trauma or fever. There was no family history of rheumatological diseases. He could not bear weight on the left side. There was no erythema, swelling, or increased warmth over the left hip, but with pain on active and passive motion. There was remarkable limitation in adduction and internal rotation of the hip. Laboratory results including complete blood count (CBC), erythrocyte sedimentation rate (ESR) and C-reactive protein (CRP) were normal. Hip radiographs (Fig. 1) revealed concentric narrowing of the left joint space with periarticular osteopenia.

He was treated initially with bed rest and non-steroidal antiinflammatory drugs (NSAIDs). At 2-week follow up, the pain was relieved but the range of motion had not improved. Physiotherapy was advised. One month later, the movements of the left hip were nearly normal. However he absconded from follow-up until one year later when the hip had eventually ankylosed.

\section{Case 2}

An 11-year old girl presented with right hip pain of one month duration. She was a healthy girl, with no previous illness or injury nor family history of rheumatologic diseases. Examination revealed antalgic gait, and restriction of internal and external rotation of the right hip. ESR, CRP and $\mathrm{CBC}$ were normal. Radiographs of the hips showed narrowing joint space of the right side with pelvic obliquity (Fig. 2).

The treatment included NSAIDs and physiotherapy. During the following months the pain was minimal, but the motion was further reduced. Seven years after the initial presentation, she was pain-free. The right hip had 20 degrees of external rotation deformity. 

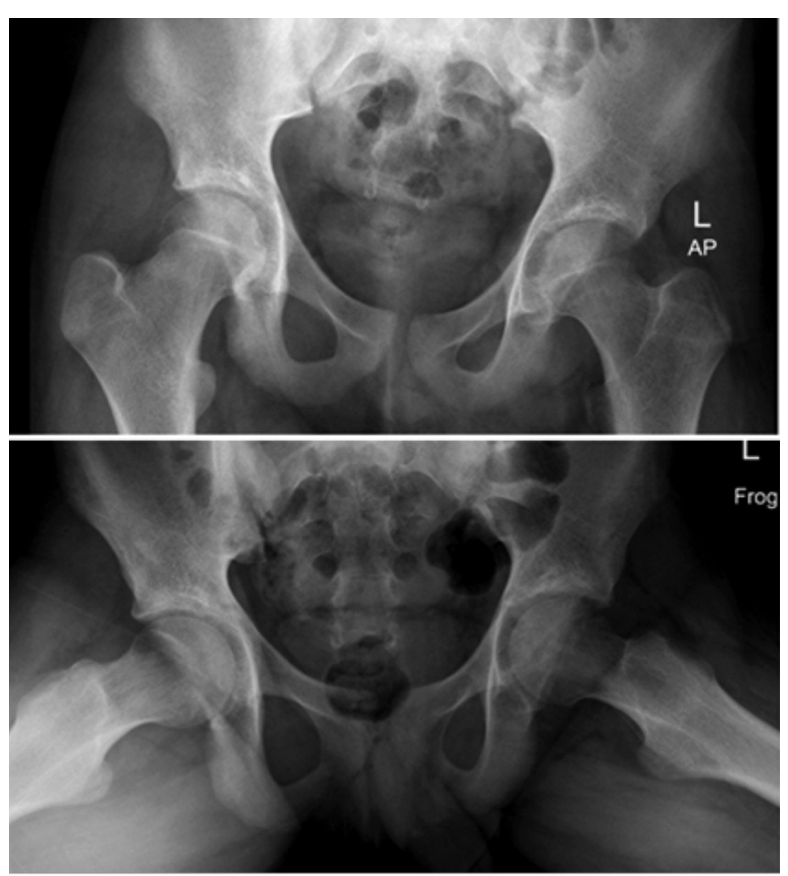

Fig. 1: (Case 1) Radiographs of both hips reveal concentric narrowing of left joint space with periarticular osteopenia.
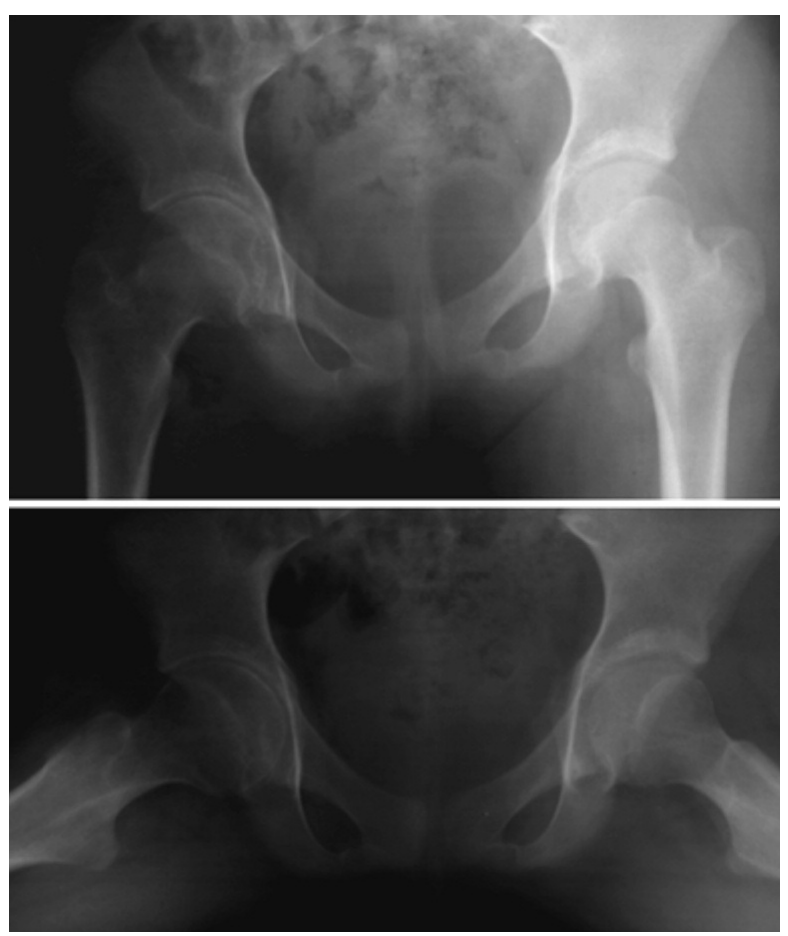

Fig. 3: (Case 3) Radiographs of both hips reveal diffuse narrowing of the right joint space with periarticular osteopenia and subchondral bone cyst.

\section{Case 3}

A 12-year old girl presented to another hospital with right thigh pain of 5 months duration. She was diagnosed as synovitis of the right hip. Several courses of NSAIDs and physical therapy failed to improve the symptoms. She then
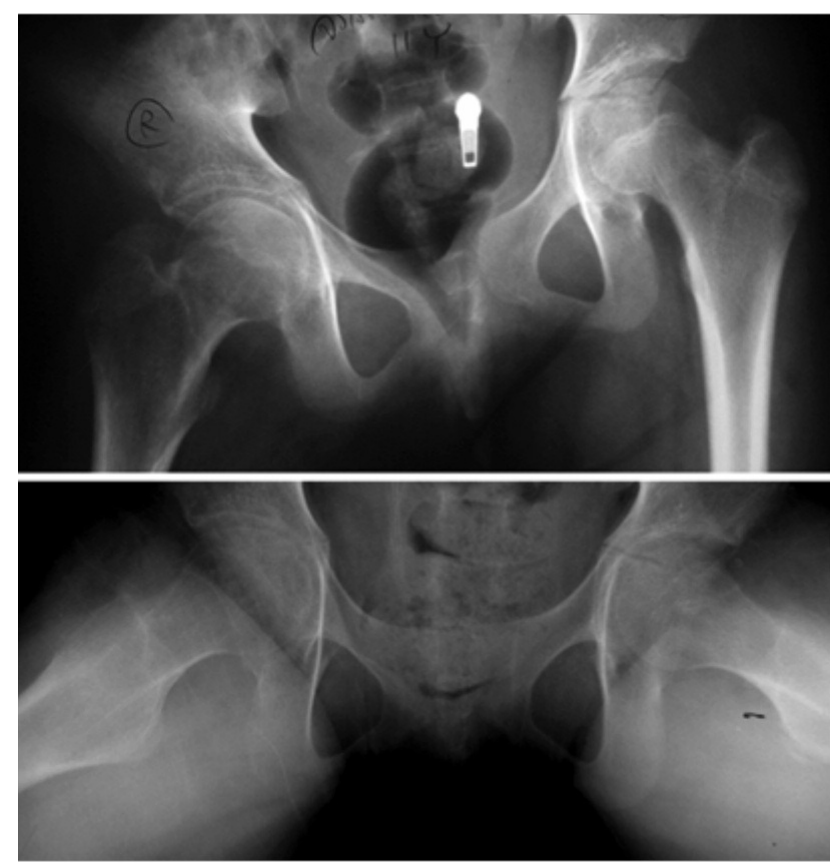

Fig. 2: (Case 2) Radiographs of both hips reveal narrowing joint space of the right hip with pelvic obliquity.

came to our hospital for further evaluation. Examination revealed right-sided antalgic gait and pelvic obliquity. There was restriction of flexion, adduction, internal and external rotations on the affected side. CBC, CRP and rheumatoid factor (RF) were normal. The ESR was slightly elevated. Tuberculin test was negative. Radiographs of the hips showed diffuse narrowing of the right hip joint space with periarticular osteopenia and subchondral bone cyst (Fig. 3). MRI revealed active inflammatory arthritis of right hip joint with patchy marrow oedema and enhancement at epiphysis of right femoral head.

She was treated initially with bed rest, traction, NSAIDs and physical therapy. However, since her symptoms did not subside and septic arthritis could not be excluded, arthrotomy of the right hip joint was performed. Muscle release for correction of abduction contracture was done simultaneously. Microbiology reports on the biopsy material showed no growth. Eight week post-operation, the range of motion was decreased. At 4-year follow up, she had normal gait. The resting position of her right hip was 15 degrees of external rotation.

\section{DISCUSSION}

ICH is characterized by the rapidly progressive destruction of articular cartilage in the hip joint resulting in premature degeneration and subsequent joint arthrosis. The aetiology is uncertain. The onset is early in the second decade of life with an approximately 6:1 female gender predominance ${ }^{2,5}$. 
There is no specific diagnostic criteria for $\mathrm{ICH}^{4}$. In clinical practice, the diagnosis is made by exclusion of other more common causes of acute or chronic monoarticular hip pain such as trauma, SCFE, septic arthritis, tuberculosis, Perthes' disease, pigmented villonodular synovitis (PVNS), synovioma and monoarticular rheumatoid arthritis. The typical clinical presentation is an adolescent female with insidious onset of hip pain, limping and contracture of the hip joint (most common in flexed, abducted and externally rotated position $)^{2,5}$.

Laboratory values are normal. ESR may be slightly raised ${ }^{5}$ ( $\leq 30 \mathrm{~mm} / \mathrm{hr}$ ). The radiographic findings are characteristic. The radiological hallmark of this condition has been described as symmetric narrowing of the hip joint space $(<3$ $\mathrm{mm}$ ), without frank osteophyte formation, in conjunction with periarticular osteopenia ${ }^{2,3,5}$. A variety of possible radiographic findings without significant pathological related has been reported ${ }^{2,3,5}$.

The three aims of treatment are pain relief, deformity correction and restoration of motion ${ }^{3}$. The treatments are mainly symptomatic and non-operative. The role of surgery is controversial. Section of tendons and muscles, capsulotomy and subtotal capsulectomy have all been performed with variable success ${ }^{2,3,5}$. Pain is the symptom that has shown the most improvement after treatment ${ }^{2}$.
In reviewing the literature approximately one hundred cases have been reported, with a few reports of more than five cases. Three patients in the present study presented with different clinical symptoms that could mimic SCFE, juvenile idiopathic arthritis (JIA) and septic arthritis. However all of them had the similar characteristic radiographic findings for $\mathrm{ICH}$. The history, physical examination, laboratory data and radiographs all supported the diagnosis of ICH. The initial treatment was conservative. Surgical treatment was performed in one patient in order to confirm diagnosis and correct deformity. At the time of follow-up ( after one, four and seven years), all of them were pain-free but with functional joint stiffness.

ICH is a rare condition and often misdiagnosed as chronic infective or inflammatory arthritis. Awareness of the existence of this disease will lead to earlier diagnosis. There are characteristic clinical and radiological features but no effective therapy to delay degenerative process of the disease. More studies are required for this condition to improve the knowledge about aetiology and pathophysiology that can be lead to effective management in the future.

\section{REFERENCES}

1. Duncan JW, Nasca R, Schrantz J. Idiopathic chondrolysis of the hip. J Bone Joint Surg Am 1979; 61(7): 1024-8.

2. Del Couz Garcia A, Fernandez PL, Gonzalez MP. Idiopathic chondrolysis of the hip: long-term evolution. J Pediatr Orthop 1999; 19(4): 449-54.

3. Korula RJ, Jebaraj I, David KS. Idiopathic chondrolysis of the hip: medium-to long-term results. ANZ J Surg 2005; 75: 750-3.

4. Povencher MT, Navaie M, Solomon DJ. Current concept review: joint chondrolysis. J Bone Joint Surg Am 2011; 93: $2033-44$.

5. Segaren N, Abdul-Jabar HB, Segaren N, Hashemi-Nejad A. Idiopathic chondrolysis of the hip: presentation, natural history and treatment options. J Pediatr Orthop B 2014; 23: 112-6. 